\title{
Molecular dynamies simulations reveal the role of five BCR-ABL kinase domain critical residues in TKIS binding
}

\author{
P. Buffa' $₫$, C. Romano², A. Pandini', P. Vigneri², F. Fraternali' \\ 'Randall Division of Cell and Molecular Biophysics, King's College of London, United Kingfom \\ ${ }^{2}$ Clinical and Molecular Biomedicine Department, University of Catania, Italy
}

\begin{abstract}
Motivations
Chronic Myeloid Leukemia (CML) is a myeloproliferative disorder characterized by a well know molecular hallmark: the BCR-ABL chimeric oncoprotein. BCR-ABL displays constitutive tyrosine kinase activity, which favours the expansion of the leukemic clone by increasing proliferation and reducing cell death. Suppression of BCR-ABL catalytic activity by Tyrosine Kinase Inhibitors (TKIs) has dramatically improved the natural history of the disease achieving unprecedented results. However, an increasing number of point mutations inside the BCR-ABL kinase domain has been associated with different degrees of resistance. Insights into the critical residues involved in the interaction between the BCR-ABL tyrosine kinase domain (TKD) and different TKIs could explain the mechanisms allowing BCR-ABL mutants to avoid kinase inhibition.
\end{abstract}

\section{Methods}

We have determined that five amino acids (E286, T315, M318, 1360, D381) are critical for Imatinib (IM) binding. Two of them (T315 and M318) are also necessary for the binding of the second generation (2G) TKI Dasatinib (DAS), while, E286, M318, 1360 and D381 are required for the interaction with the third generation (3G) inhibitor Ponatinib (PON). Only one (315) of these five positions shows amino acidic substitutions in TKI-resistant patients. We used Modeller-v9.8 to generate in silico mutants that were predicted to maintain catalytic activity. We subsequently performed Molecular Dynamics (MD) simulations on these mutants in a simulated aqueous environment using the GROMACS package with AMBER force field (ffamber99sb), obtaining 50ns of data collection for each system. We also carried out MD simulations of these mutants in complex with IM, DAS, and PON. The simulation trajectories were analyzed with both GROMACS analysis packages and Principal Component Analysis (PCA) in order to identify large-scale motions defining the functional dynamics of both wild-type and mutated BCR-ABL.

\section{Results}

We demonstrated that the conservative I360T mutation displaces the helix- $\alpha \mathrm{C}$ located in the $\mathrm{N}$-lobe of the BCR-ABL tyrosine kinase domain, moving away E286 from the catalytic pocket. This residue is one of the critical amino acids for IM binding. When we docked IM inside the pocket of the BCR-ABL TKD displaying the helix displaced and performed further 50ns of MD simulation we found that the C-helix moves back towards the catalytic pocket, possibly under the attractive electrostatic field generated by the drug. We also found that IM restores three of the five original h-bond interactions warrantying IM binding. This result is in agreement with experimental data showing that BCR-ABLI360T is inhibited by IM. Our data also confirm the efficacy of the $3 G$ inhibitor PON on CML patients failing IM and $2 G$ TKIs because of its ability to maintain the same interactions showed when the drug inhibits BCR-ABL wild-type. These findings could potentially be extended to further protein kinases, thus contributing to the design of other TKls targeting different protein kinases contributing to the pathogenesis of additional neoplastic diseases. 